\title{
Anglais de spécialité et pédagogie differenciée dans les BTS : le cas des causatifs
}

Jacques Coulardeau

\section{CpenEdition}

Journals

Édition électronique

URL : http://journals.openedition.org/asp/4383

DOI : 10.4000/asp.4383

ISSN : 2108-6354

Éditeur

Groupe d'étude et de recherche en anglais de spécialité

Édition imprimée

Date de publication : 1 mars 1993

Pagination : fr

ISSN : 1246-8185

Référence électronique

Jacques Coulardeau, «Anglais de spécialité et pédagogie differenciée dans les BTS : le cas des causatifs », ASp [En ligne], 1 | 1993, mis en ligne le 06 mai 2014, consulté le 03 mai 2019. URL : http:// journals.openedition.org/asp/4383; DOI : 10.4000/asp.4383

Ce document a été généré automatiquement le 3 mai 2019.

Tous droits réservés 


\title{
Anglais de spécialité et pédagogie differenciée dans les BTS : le cas des causatifs
}

\author{
Jacques Coulardeau
}

1 Nous allons tenter de prendre le sujet du colloque à bras le corps: «La formation en anglais de spécialité en fonction du niveau de compétence des étudiants dans les disciplines principales »

2 Dans les BTS les choses se passent un peu différemment car nous travaillons en classe homogène, officiellement, et non en unités de valeur ouvertes à tous, indifféremment de leur niveau dans le cursus. Dans ces classes officiellement homogènes apparaissent des groupes de niveau en anglais qui ne recoupent pas ou sont même parfois l'image inversée des groupes de niveau en enseignement principal.

3 L'anglais est aussi une matière refuge pour certains étudiants qui veulent avoir un ballon d'oxygène non technique.

4 La question est donc pour nous : "Comment différencier l'enseignement dans une classe homogène, en tenant compte des visées et des motivations multiples et bien sûr du niveau dans les matières principales qui libèrent plus ou moins de temps pour les matières d'enseignement général qui, tout en étant ancillaires, se doivent, du moins en ce qui concerne l'anglais, d'avoir leurs propres objectifs ».

\section{Les besoins d'anglais d'un BTS audiovisuel}

\subsection{Point de vue de l'administration}

5 C'est un point de vue réductif. L'administration a tendance à considérer que ces étudiants et les techniciens qui sortiront de ces classes n'ont qu'un besoin exclusivement passif: savoir lire que ce soit sur papier ou sur écran. D'autre part, nous avons une seconde 
tendance réductrice: assimiler une connaissance exclusivement et étroitement spécialisée qui est réduite à du lexique. L'objectif est clair : savoir lire des instructions papier ou machine du seul champ technique concerné : notices techniques et instructions de fonctionnement, ce qui exclut corrélativement toute approche un peu riche de la syntaxe de cet anglais, syntaxe qui est réduite aux fonctions de base de l'anglais telles qu'elles sont définies dans le second cycle secondaire technique.

\subsection{Ce point de vue est irréaliste}

D'abord du fait du poids de plus en plus lourd de l'informatique dans tous les champs industriels de l'anglais de spécialité et, même bien au-delà, quand on sait que toute la littérature informatique qui se tient un tant soit peu est en anglais. Les logiciels les plus avancés ne sont pas francisés et les livres de présentation et d'étude les plus pertinents sont en anglais, un anglais très américanisé et très lourd syntaxiquement. Faire l'économie de cet anglais de l'informatique c'est se condamner à produire des techniciens qui ne seront pas performants dans leur domaine technique sauf formation complémentaire.

Ensuite c'est négliger totalement l'intercommunicationnel omniprésent dans la vie de l'entreprise de notre époque: une entreprise nécessairement multinationale dans un système économique globalisé au plus haut point. Nous voyons la mise en place de ce que MacLuhan appelait le « village global » à une vitesse qui défie l'imagination.

Cela veut dire que l'on négligerait, si on suivait la ligne officielle, que le technicien doit aussi savoir parler à la machine qui ne reçoit dans les domaines de pointe que des ordres écrits en anglais. C'est négliger aussi la communication homme-homme qui passe par trois vecteurs: les réseaux informatiques, le téléphone, et bien sûr les conférences, briefings et autres rencontres de discussion au niveau de la conception, de la production, de l'exploitation et de la commercialisation des produits.

9 C'est également négliger que dans la phase actuelle de développement économique, une équipe technique un tant soit peu développée se doit d'intégrer des étrangers et un technicien un tant soit peu avancé se doit d'être capable de s'intégrer à des équipes étrangères et de fonctionner en terres étrangères, et en premier lieu dans des terres où l'anglais est la langue véhiculaire. Ce sont donc les quatre compétences cardinales qu'il faut développer chez le technicien moyen ou supérieur : la compréhension tant écrite que orale et l'expression tant écrite qu'orale. Et n'oublions pas que dès qu'une machine entre en jeu, il n'est plus possible de faire la moindre faute car la latitude de tolérance des machines à l'égard des fautes est des plus réduites.

Mais c'est aussi oublier la formidable responsabilité du technicien moyen et supérieur. Un technicien manie des machines et des processus de production qu'il peut par une erreur de compréhension ou d'expression mettre dans une impasse technique ou économique pouvant causer des dégâts extrêmement importants et chiffrables en sommes monétaires colossales. De plus, par une erreur de manœuvre due à une erreur de compréhension d'un message ou d'une instruction, le technicien peut mettre en péril la vie de ses camarades de travail, ou simplement de personnes innocentes dans le public. La responsabilité civile du technicien est engagée. Mais plus encore celle de la personne qui a mis sur une machine ce technicien, sans s'assurer qu'il avait un niveau suffisant d'anglais pour ne pas causer des accidents. Ce dernier point d'ailleurs est la seule défense du technicien face à sa responsabilité dans l'accident. 


\section{Les principes du chapitre sur les causatifs}

11 en chantier pour le rattrapage linguistique des BTS industriels. Les principes qui nous ont guidés dans son écriture sont les suivants.

\subsection{S'appuyer prioritairement sur l'anglais de spécialité}

En priorité ne veut pas dire exclusivement, mais cela veut dire que nous visons une bonne description de cet anglais de spécialité industriel, particulièrement de sa base informatique, et que nous avons fait un gros travail d'exploration de corpus et de documents pour parvenir à une description beaucoup plus fine que tout ce qui existe. Mais cela n'est pas exclusif car les besoins linguistiques des techniciens industriels dépassent énormément les seules applications industrielles. Nous avons aussi intégré des éléments d'anglais général, à l'exception de l'anglais littéraire. Pour nous, partir de l'anglais de spécialité est le moyen d'intéresser davantage la majorité de nos étudiants.

\subsection{Nous partons d'un principe totalisant}

Nous voulons présenter toute la matière, tout l'anglais de spécialité et non pas des bribes qui laissent en panne des éléments, peut-être plus rares, mais qui peuvent causer des dégâts monumentaux le jour où ils interviennent.

\subsection{Nous posons comme principe de base un principe explicatif}

Nous ne voulons pas simplement que l'étudiant soit capable de reconnaître une structure et de mettre en face sa correspondance française. Nous voulons qu'il comprenne ce qui se passe quand une telle structure est en jeu, ce qui se passe dans la structure elle-même et ce qui se passe dans son utilisation. Nous pensons qu'en appeler à la tête compréhensive de l'étudiant, c'est aussi se donner le maximum de chances de réussir à travailler avec tous et amener le maximum d'étudiants au niveau visé.

\subsection{Le principe de l'auto-apprentissage}

15 Nous pensons qu'un étudiant de ce niveau doit être capable d'apprendre par lui-même. Il ne suffit pas de le décréter, encore faut-il lui donner des outils pour ce travail. Notre approche vise donc à donner toujours la correction de la moitié des exercices pour que l'étudiant puisse après son étude des présentations grammaticales, affronter le travail d'application par lui-même. Si nous ne donnons pas à cet étudiant les moyens de travailler par lui-même, nous l'infantilisons. Si nous l'infantilisons, nous nous condamnons à échouer avec un nombre très grand d'étudiants. L'auto-apprentissage est la garantie que l'étudiant peut, s'il le veut, marcher de son propre pas.

Une longue discussion s'est engagée sur ce principe. Il ressort de cela que l'autoapprentissage est difficile. D'abord il y a peu d'outils disponibles et il faut les recenser dans un premier temps. Ensuite il faut une motivation importante de la part des apprenants : la motivation existe dans les classes de BTS du fait de la sélection. Elle existe 
encore plus dans les classes d'adultes dans le cadre du $1 \%$. Cependant on s'aperçoit que les étudiants en font plus ou moins sans que l'on sache vraiment pourquoi certains en font plus que d'autres. Le rapport peut aller d'un à cinq selon mon expérience. Et encore j'ai toujours imposé un minimum: par exemple en travail au téléphone, j'ai toujours donné un document à un étudiant à charge pour lui de le préparer et d'être au téléphone tel jour à telle heure pour m'en parler et le discuter, le premier document étant obligatoire, les suivants étant volontaires. Mais une autre difficulté semble apparaître : cette stratégie nous amène à introduire des rapports différents avec les étudiants pour les motiver davantage. Ces rapports plus détendus, plus «copinants» sont peut-être dangereux du fait des transferts qu'ils manipulent, mais surtout du fait des problèmes qu'ils peuvent créer autour, dans d'autres classes où les rapports traditionnels sont seuls de mise. On a alors des difficultés avec le reste de l'équipe, ou avec certains membres de l'équipe qui considèrent que leurs problèmes sont causés par les rapports particuliers que nous entretenons avec les étudiants. Cela est vrai en partie, mais cette explication de leurs difficultés est plus que facile.

Ceci étant on constate que l'auto-apprentissage marche avec certains, bien ou moins bien, mais ne marche pas avec d'autres et on est totalement incapable de dire pourquoi. On ne peut que constater.

Il est ressorti fortement dans la discussion que l'auto-apprentissage ne peut fonctionner, ne peut avoir une quelconque efficacité que si le professeur est derrière et s'il vérifie le travail et demande des rapports réguliers de progression. C'est là à la fois la condition du fonctionnement de cette technique didactique et sa propre perversion puisque c'est la négation même de l'entière responsabilité de l'apprenant. En ce qui me concerne, je demande des bilans qui repèrent les difficultés non résolues et donc qui apportent des aides supplémentaires lorsque l'auto-apprentissage rencontre une limite. On a parlé dans le débat de transfert des moments de l'apprentissage plus que de remplacement d'une méthode par une autre. Le professeur intervenant en aval de l'apprentissage et non plus en amont. Son rôle en amont étant de préparer les matériaux et non plus de les présenter. L'idéal semble être l'existence d'un lieu d'auto-apprentissage avec des "tuteurs" disponibles sur ce lieu, ce qui permet un guidage plus immédiat de la procédure.

La discussion a enfin mis en avant avec force l'idée que l'auto-apprentissage est une bonne méthode de gérer des groupes hétérogènes pour les faire travailler à la fois individuellement et collectivement, mais sous la responsabilité de rendre des travaux qui sont dûment jugés avec trois niveaux possibles : acceptables, à reprendre sur certains points ou à refaire entièrement.

\subsection{Le travail du maître}

Il a trois fonctions principales. D'abord vérifier si la compréhension des explications est bien passée. Sinon il doit les reprendre et s'assurer que tous ont compris le b-a-ba du point de grammaire. Ce travail n'est pas aussi simple qu'il y paraît. Il pose de nombreux problèmes, et en particulier comment mener de front des étudiants qui ont compris et des étudiants qui n'ont pas compris. Là tout est à inventer, car depuis une trentaine d'années on a interdit la grammaire et le travail structural, ce qui fait qu'on a négligé la didactique de ce travail structural. Donnons quelques pistes: doit-on utiliser ceux qui comprennent pour expliquer aux autres? Doit-on mettre des groupes de travail hétérogènes en place pour l'auto-apprentissage? Doit-on laisser les étudiants autogérer 
ce problème à leur convenance, en se disant que qui dit autogestion dit aussi désordre dans l'affrontement des problèmes?

Ensuite approfondir, c'est à dire principalement répondre à des questions qui n'ont pas trouvé réponse dans le texte, ou des questions qui sont apparues dans certains esprits au contact du texte. Encore une fois, l'absence de réflexion théorique pendant trop longtemps sur le terrain d'application fait que l'on est désemparé pour prévoir toutes les questions qui peuvent se soulever. Nous devons faire acte de modestie mais aussi de réflexion, si possible collective, devant ce qui surgit.

Enfin poursuivre le travail, c'est-à-dire finir les exercices. Cela permet de vérifier s'il y a bien eu compréhension, si la capacité manipulatoire a bien été acquise. Cela risque, à la pratique, de remettre en cause certaines présentations et certaines explications car inefficaces ou bien poussant l'étudiant dans des voies de garage.

Comme on le voit, le rôle du professeur n'est plus de transmettre un savoir, mais essentiellement de vérifier son acquisition et d'approfondir cette acquisition. C'est là un rôle neuf pour le professeur d'anglais dans le domaine technique industriel.

\subsection{Les causatifs en anglais de spécialité}

24 Nous avons fait, pour écrire ce chapitre un travail de corpus : nous avons lu en entier le numéro de mai 1991 de Byte et avons relevé toutes les formes qui ont rapport avec la notion de causation. Cela nous donne en premier lieu des données chiffrées (voir tableau 1).

Tableau 1

\begin{tabular}{|l|l|l|}
\hline make & 100 & $28,25 \%$ \\
\hline have & 24 & $6,77 \%$ \\
\hline let & 21 & $5,93 \%$ \\
\hline permissifs & 185 & $52,26 \%$ \\
\hline autres & 24 & $6,77 \%$ \\
\hline Total & 354 & $100,00 \%$ \\
\hline
\end{tabular}

Cet ensemble de données chiffrées montre déjà que nous ne pouvons réduire l'approche des causatifs aux seuls causatifs « cardinaux » que sont make et have. Il est indispensable de faire une présentation plus large qui s'ouvre largement sur les causatifs permissifs, à savoir let, help, permit, enable, allow. On sait de plus que ces trois derniers posent de gros problèmes aux Francophones car ils se construisent normalement avec un « complément » intermédiaire, ce qui n'est pas le cas en français.

Plus encore, notre approche fait apparaitre non pas deux structures de base, la structure active, qui se construit avec l'infinitif sans TO, et la structure passive, qui se construit avec le participe passé, mais une troisième, la structure médiane qui se construit avec la 
forme en V-ing. Cela fait jouer à get un rôle particulier car il admet les trois constructions. Mais cela est aussi le cas de have.

... to have someone else deal with it.

... we had it set up.

... we had it running.

... to get your network operating system to understand TCP/IP.

... we can get the plumbing fixed.

... to get the product running. certain niveau de conscience de ces différences qu'il est capable de les dominer. Ceux qui réussissent sans passer en classe par ces explications, sont ceux qui sont capables de les reconstruire dans leur tête sans l'aide d'un professeur. Ils sont la minorité et se retrouvent rarement dans les filières industrielles. On pourrait ensuite discuter les exercices un par un. Mais là n'est pas mon objet pour le moment.

\section{Performance dans la matière principale et stratégie didactique}

\subsection{L'anglais}

Nous avons trois niveaux qui se dégagent dans nos classes de BTS.

D'abord les très faibles qui nécessitent beaucoup de rattrapage et donc une pédagogie de la remise en ordre du plus simple, de la réacquisition de ce plus simple. 

début, car ils ont déjà travaillé sur ces matériels. Mais il leur manque des enseignements généraux pour valoriser leur avantage et surtout passer de la simple manipulation ou réparation à l'utilisation, l'exploitation, voire des techniques complexes d'utilisation comme le montage. le plus souvent touché à l'informatique ou à des techniques proches comme l'électrotechnique ou l'électronique. Ils sont capables le plus souvent d'aller très vite dans l'acquisition des techniques de base. l'informatique. Ils attendent des cours très intensifs pour aller très vite, comme leurs capacités générales le leur permettent. Ils sont souvent un peu déçus à cause des rythmes trop lents et donc frustrants.

Comme on peut le remarquer, il n'y a pas vraiment recoupement entre ces trois groupes et les trois groupes de l'anglais. Ou du moins, il n'y a que recoupement partiel. Il y a recoupement dans le haut de l'échelle, mais il n'y a pas correspondance dans le bas de l'échelle. D'autre part, les étudiants du haut de l'échelle ont tendance à vouloir faire déraper les cours d'anglais de l'anglais de spécialité vers un enseignement littéraire, culturel ou artistique.

Il est cependant intéressant de remarquer que les capacités dans les matières principales ont une incidence directe sur le fonctionnement des classes : lorsque nous abordons des termes techniques et particulièrement des termes de pointe qui n'ont pas encore trouvé place dans des dictionnaires monolingues ou bilingues, on peut, c'est notre travail, donner ce que notre « intuition morphosyntaxique " nous amène à penser de ce que ces 
termes signifient, et on s'appuie sur les meilleurs dans les matières techniques pour éventuellement rechercher un terme équivalent en français. C'est un travail difficile, car nous n'avons aucun moyen de vérifier la justesse de cette information. Il s'agit de faire confiance, et surtout, avant de faire confiance, de susciter un véritable esprit de collaboration et de recherche. Il va de soi que nous ne pouvons pas travailler tout le temps avec des professionnels, et que même eux ont souvent des difficultés pour suivre nos demandes. Nous aurions besoin de travailler avec des professionnels anglophones dans un pays anglophone. Poser cette exigence c'est montrer que cela est impossible. Là on a vraiment la possibilité sinon la nécessité d'utiliser les meilleurs dans les matières techniques pour notre travail linguistique.

\subsection{Une stratégie}

Pour les faibles, nous avons proposé des cours de rattrapage pour adultes qui sont des cours d'initiation de bas niveau pour adultes dans le cadre du GRETA. Nous avons ajouté un manuel d'auto-apprentissage, à savoir English for the Telecommunication Industry (Comfort \& Revell 1986). Les étudiants doivent faire à leur rythme les travaux de ce manuel et le professeur vérifie régulièrement ce qu'il en est et essaie de résoudre les difficultés qui apparaissent. Ces étudiants étant issus de Bacs Pros peuvent faire ce travail supplémentaire car ils n'ont pas beaucoup de choses à faire pendant la première année en matières techniques où il s'agit d'une initiation à ces techniques, initiation dont ils sont largement dotés. Dans ces matières, il s'agit pour eux plus de changer de perspective, de l'entretien à l'exploitation, plutôt que d'apprendre beaucoup de choses neuves.

La discussion a fait apparaître que l'auto-apprentissage ne peut fonctionner que dans la mesure où il y a un suivi obligatoire pour les étudiants. S'il n'y a pas ce suivi, il y a rapidement abandon de la procédure et donc simplement auto-abandon à son niveau faible.

Pour les forts, nous avons proposé des travaux de composition et de réflexion sur divers sujets élargissant la palette de l'anglais de spécialité : comptes rendus de films, études et comptes rendus d'œuvres plus littéraires (Allan Ginsberg, Stephen King, Clive Barker ou des articles du Cinematographer, revue américaine). Tous les étudiants doivent faire le travail, mais la palette est suffisante pour que chacun choisisse en fonction de son niveau, et le professeur se doit de moduler la notation en fonction de l'étudiant, niveau qui se reflète largement dans le traitement du sujet, du résumé factuel plat, à une véritable synthèse commentée.

Nous pouvons remarquer que dans ces travaux, étrangement, mais peut-être pas aussi étrangement que cela pourrait en avoir l'air, c'est Stephen King qui remporta largement l'agrément de la majorité des étudiants, que ce soit ses films (Christine) ou que ce soit la nouvelle proposée (I'm the Doorwav).

Pour tous une stratégie de révision-approfondissement-élargissement s'appuyant principalement sur un travail en grammaire et en structure de la langue de spécialité. Une stratégie d'auto-apprentissage qui permet des rythmes différents. Une stratégie pédagogique du maître qui reprend, remet en perspective et élargit sans cesse, que ce soit à partir de la grammaire ou à partir de textes d'anglais de spécialité. 


\section{Conclusions}

50 Nous avons deux types de conclusions. D'abord, nous devons adopter une visée explicative qui a l'avantage de remettre tout le monde au point un, au point de départ et au pied du mur avec des chances beaucoup plus égales. La compréhension ne dépend pas tant du savoir antérieur que des capacités intellectuelles des étudiants. L'égalité est alors beaucoup plus grande, étant donnée la très forte sélection de ces étudiants, ce qui assure un bon partage des capacités intellectuelles.

51 Ensuite, nous devons diversifier le travail, et cela prend la forme d'une pédagogie différenciée. Différencier les rythmes en faisant une pédagogie à la carte par diverses actions qui laissent l'étudiant rechercher son rythme et le suivre: manuels d'autoapprentissage ou bien formes d'intervention où le volontariat permet de prendre plus ou moins de matière proposée et donc d'aller plus ou moins vite selon le courage que l'on a ou le temps dont on dispose.

Différencier le contenu en donnant un exercice dont la forme est la même pour tous, mais dont le sujet va différer dans sa matière, d'où la possibilité pour certains de choisir le sujet le plus difficile, et pour d'autres de prendre le sujet le plus simple. L'étudiant va rechercher ce qui lui convient le mieux. Il y a un risque de paresse, c'est sûr, mais cela révèle plus la motivation de l'étudiant qu'autre chose. La motivation, certes, ça se construit et le maître joue un rôle capital, mais ça ne s'impose pas à un étudiant. À chacun de choisir son profil d'étude, même si certains sont suicidaires. À nous de le leur rappeler et d'utiliser la notation pour marquer notre position.

Différencier par l'ouverture. Le professeur d'anglais ne peut pas s'enfermer dans sa matière, même si c'est l'anglais de spécialité. Il doit s'ouvrir au reste de l'équipe pédagogique, dans la mesure du possible. Il doit aussi s'ouvrir au champ professionnel concerné et s'imposer un gros travail de découverte de l'anglais dans ce champ professionnel: lecture régulière de magazines spécialisés en anglais, et tentative de comprendre ce dont ces magazines parlent. Il doit s'ouvrir sinon à la connaissance complète et opérationnelle de ces techniques, du moins à une connaissance minimale sans laquelle il ne peut même pas comprendre la langue qu'il tente d'enseigner, sans compter qu'il se trouverait souvent en porte à faux s'il ignorait tout ce dont il prétend parler. Modestie et connaissance sont les deux mamelles du professeur en anglais de spécialité. Il doit aussi s'ouvrir à une autre dimension fort importante dans les métiers audio-visuels : les pratiques créatrices. Certes il ne peut pas être auteur ou plasticien dans tous les domaines de l'art et de la création. Mais il se doit d'être un connaisseur avisé au moins dans certains domaines de ces pratiques culturelles ou artistiques. Ne pas savoir quelque chose n'est pas grave. Ne rien savoir est très dangereux. Ceci n'est d'ailleurs pas vrai que du professeur de langue.

Cela me donne le mot de la fin. Certes il ne sera jamais à même d'en apprendre aux meilleurs étudiants dans les champs techniques, mais il doit être capable de soutenir une discussion générale sur certains enjeux de ces champs techniques, enjeux globaux (économiques, sociaux, idéologiques, historiques...) ou enjeux techniques (pour ne prendre qu'un exemple, il doit saisir la dimension absolument nouvelle que la technique de la réalité virtuelle représente dans l'audiovisuel, même s'il n'est pas capable d'en discuter ou d'en comprendre les éléments les plus techniques). 
55 En fait le professeur de langue se doit d'acquérir un savoir et ce sont les étudiants les plus avancés dans les matières techniques qui sont les plus à même et les plus volontaires pour lui donner cette connaissance. Et, disons-le franchement, être le professeur du professeur est pour eux quelque chose de fondamentalement gratifiant. Et cela donne aux moins avancés l'exemple de la modestie et de la nécessaire acquisition du savoir qui est un processus jamais achevé.

\section{BIBLIOGRAPHIE}

Comfort, Jeremy \& Rod Revell. 1986. English for the Telecommunication Industry. Oxford : Oxford University Press

\section{AUTEUR}

\section{JACQUES COULARDEAU}

Lycée Jean Rostand Roubaix, UA 1030 CNRS Lille. dondaine@orange.fr 